\title{
Aumento de Óbitos Domiciliares devido a Parada Cardiorrespiratória em Tempos de Pandemia de COVID-19
}

\author{
Increased Home Death Due to Cardiopulmonary Arrest in Times of COVID-19 Pandemic
}

Nathalia Sernizon Guimarães, ${ }^{1 \oplus}$ Taciana Malheiros Lima Carvalho, ${ }^{2} \oplus$ Jackson Machado-Pinto, ${ }^{2}$ Roger Lage, ${ }^{2 \oplus}$ Renata Mascarenhas Bernardes, ${ }^{2}$ Alex Sander Sena Peres, ${ }^{2}$ Mariana Amaral Raposo, ${ }^{3 @}$ Ricardo Machado Carvalhais, ${ }^{4}$ Renan Avelino Mancini, ${ }^{4 \oplus}$ Gabriella Yuka Shiomatsu, ${ }^{4 \oplus}$ Bruna Carvalho Oliveira, ${ }^{4 \oplus}$ Valéria de Melo Rodrigues, ${ }^{4 \oplus}$ Maria do Carmo Barros de Melo, ${ }^{4}$ Unaí Tupinambás ${ }^{4}$

Universidade Federal de Ouro Preto, ${ }^{1}$ Ouro Preto, MG - Brasil

Prefeitura Municipal de Belo Horizonte, ${ }^{2}$ Belo Horizonte, MG - Brasil

Universidade Estadual de Campinas, ${ }^{3}$ Campinas, SP - Brasil

Universidade Federal de Minas Gerais, ${ }^{4}$ Belo Horizonte, MG - Brasil

\section{Resumo}

Fundamento: As doenças cardiovasculares constituem um grupo importante de causas de morte no Brasil. As doenças isquêmicas do coração são as principais causas de parada cardiorrespiratória, levando a um impacto na mortalidade devido às doenças cardiovasculares no sistema de saúde.

Objetivo: Avaliar o número de óbitos domiciliares por parada cardiorrespiratória notificados pelo Serviço de Atendimento Móvel de Urgência (SAMU) em março de 2018, 2019 e 2020.

Métodos: Trata-se de um estudo observacional realizado a partir da análise de dados de mortalidade por parada cardiorrespiratória de cidadãos atendidos pelo SAMU em Belo Horizonte, Minas Gerais, Brasil. Foram analisadas as características sociais e clínicas e as informações de ocorrência. Foi avaliada a taxa de mortalidade por parada cardiorrespiratória em relação ao número total de atendimentos. Foi considerado um nível de significância de $95 \%$.

Resultados: Houve um aumento nos óbitos domiciliares por parada cardiorrespiratória em março de 2020, em comparação com março de 2018 ( $p<0,001$ ) e março de 2019 ( $p=0,050)$. Dos óbitos relatados em 2020, 63,8\% dos pacientes tinham 60 anos ou mais; $63,7 \%$ das ocorrências foram à tarde e aproximadamente $87 \%$ dos casos de parada cardiorrespiratória notificados apresentavam comorbidades clínicas, com hipertensão arterial sistêmicas e parada cardíaca correspondendo a 22,87\% e 13,03\% dos casos relatados, respectivamente. A maioria da amostra avaliada deste estudo não teve acompanhamento médico (88,7\%).

Conclusão: Considerando o aumento do número de óbitos, sugerimos reflexões e reajustes quanto ao monitoramento das doenças crônicas não transmissíveis durante a pandemia, bem como melhorias na vigilância dos óbitos. (Arq Bras Cardiol. 2021; 116(2):266-271)

Palavras-chave: COVID-19; Betacoronavírus; Pandemia;Parada Cardíaca; Óbitos; Serviços Médicos de Emergência.

\section{Abstract}

Background: Cardiovascular diseases constitute an important group of causes of death in the country. Ischemic heart diseases that are the main causes of cardiopulmonary arrest, leading to an impact on the mortality of the cardiovascular diseases in the health system.

Objective: Assess the number of home deaths by cardiopulmonary arrest notified by the Mobile Emergency Medical Service (SAMU) in March 2018, 2019 and 2020.

Methods: Observational study carried out from the analysis of cardiopulmonary arrest mortality data of citizens assisted by SAMU in Belo Horizonte, Minas Gerais, Brazil. Social and clinical characteristics and occurrence information of the patients were analyzed. The mortality rate due to cardiopulmonary arrest in relation to the total number of attendances was assessed. A significance level of 95\% was considered.

Results: There was increase of home deaths due to cardiopulmonary arrest in March 2020 compared to March 2018 (p<0.001) and March $2019(p=0.050)$. Of the deaths reported in 2020, 63.8\% of the patients were aged 60 years or older, $63.7 \%$ of the occurrences were performed in the afternoon and approximately $87 \%$ of the cardiopulmonary arrest notified had associated clinical comorbidities, with systemic arterial hypertension and heart failure represented by $22.87 \%$ and $13.03 \%$ of the reported cases, respectively. The majority of the evaluated sample of this study did not have any medical care follow-up (88.7\%).

Correspondência: Nathalia Sernizon Guimarães •

Universidade Federal de Ouro Preto - Rua Dois, CEP 35400.000, Campus Morro do Cruzeiro, Ouro Preto, MG - Brasil

Artigo recebido em 28/05/2020, revisado em 09/08/2020, aceito em 12/08/2020

E-mail: nasernizon@gmail.com

DOI: https://doi.org/10.36660/abc.20200547 
Conclusion: Considering the increase in the number of the deaths, we suggest reflections and readjustments regarding the monitoring of chronic non-transmissible diseases during a pandemic, as well as improvements in death surveillance. (Arq Bras Cardiol. 2021; 116(2):266-271)

Keywords: COVID-19; Betacoronavirus; Pandemics; Heart Arrest; Deaths; Emergency Medical Services.

Full texts in English - http://www.arquivosonline.com.br

\section{Introdução}

O Serviço de Atendimento Móvel de Urgência (SAMU) representa o componente móvel de emergência normativamente instituído pelo Sistema Único de Saúde (SUS). Integrante da Rede de Urgência e Emergência desde 2003, o SAMU é atualmente um serviço público de assistência que atua com o objetivo de atendimento préhospitalar móvel no SUS. Além disso, este serviço transporta pacientes para hospitais privados, sendo um importante componente da admissibilidade de pacientes da rede privada de saúde. ${ }^{1,2}$

O SAMU é composto por centrais de regulação e equipes de ambulâncias, compostas por médicos e enfermeiras. De acordo com as diretrizes preconizadas pelo SUS, qualquer cidadão pode solicitar atendimento móvel pré-hospitalar por meio do acesso gratuito ao telefone, ligando para o número 192. Na central de regulação, um operador de telefone identifica o paciente e o local da ligação e transfere o atendimento ao médico regulador, que pode orientar o paciente por telefone ou ligar para a equipe de assistência para atender à solicitação do usuário. Todas as etapas do atendimento são registradas com o consentimento de ambas as partes, profissionais e usuários. ${ }^{1}$

As equipes de ambulâncias são compostas por unidades de suporte básico, nas quais estão presentes um técnico de enfermagem, motoristas e enfermeiros, e unidades de suporte avançado, uma ambulância com mais recursos tecnológicos e a presença de médico e enfermeiro. A depender das necessidades regionais, as ambulâncias são caracterizadas por motocicletas, barcos ou sistema aeromédico composto por helicóptero ou avião. ${ }^{3}$

As doenças cardiovasculares (DCV) constituem atualmente um importante grupo de causas de óbito no Brasil e no mundo. De acordo com a Sociedade Brasileira de Cardiologia, até o primeiro dia de julho, as DCV causaram mais de 198.000 óbitos entre os brasileiros em 2020. ${ }^{4}$ Estas doenças incluem as doenças isquêmicas do coração, que são as principais causas de parada cardiorrespiratória (PCR).

Segundo o Cardiômetro, indicador do número de óbitos por DCV, criado pela Sociedade Brasileira de Cardiologia, entre 2004 e 2014, as doenças isquêmicas constituíram o grupo de causas cardiovasculares com maior prevalência de eventos de óbito por DCV. ${ }^{4}$ Os dados na literatura a respeito da incidência de PCR no Brasil são escassos, sendo observado o impacto desse evento na mortalidade dos indivíduos. ${ }^{5-7}$

Neste contexto, este estudo tem como objetivo descrever o número de óbitos domiciliares por PCR notificados pelo SAMU no município de Belo Horizonte em 2020 e comparar os óbitos domiciliares por PCR em março de 2020 em relação a março de 2018 e março de 2019.

\section{Métodos}

Este estudo faz parte do serviço de notificação do SAMU de Belo Horizonte, Minas Gerais, Brasil, e se refere aos dados coletados em março de 2018, março de 2019 e março de 2020. As notificações foram selecionadas a partir do processamento manual de fichas referentes ao atendimento total das equipes no período previamente determinado. Não foram estabelecidos critérios de exclusão para avaliação de usuários/óbitos. Assim, a amostragem foi realizada por conveniência, contemplando todas as notificações cadastradas no serviço nos períodos descritos.

Trata-se de um estudo observacional retrospectivo, realizado a partir da análise de dados primários de mortalidade por PCR em cidadãos atendidos pelo SAMU em Belo Horizonte.

\section{Análise Estatística}

Foram coletadas a idade, as características de ocorrência (dia do mês e hora do dia) e as características clínicas (causa da PCR, acompanhamento médico e comorbidades associadas) e foi calculada a taxa de mortalidade de acordo com o sistema de notificação do SAMU.

Os dados foram coletados pelos pesquisadores do serviço e, subsequentemente, submetidos à análise descritiva. A análise descritiva das variáveis foi realizada por meio da distribuição de frequências e números absolutos das variáveis categóricas. Foram estimados a prevalência dos desfechos e os intervalos de confiança de $95 \%$ para a população.

Para análise dos dados, foi utilizado o programa estatístico público e gratuito OpenEpi ${ }^{\circledR}$. As variáveis categóricas foram analisadas por meio da distribuição de frequência e comparadas pelo teste qui-quadrado. O nível de significância foi definido em $95 \%(p<0,05)$

\section{Resultados}

Foram registrados 1.662 óbitos pelo SAMU nos meses de março de 2018 ( $n=563), 2019(n=494)$ e 2020 ( $n=$ 605). Nesse período (março), o SAMU registrou 919 óbitos domiciliares por PCR nos anos de 2018, 2019 e 2020, distribuídos em 260 óbitos em março de 2018 (28,3\%), 283 óbitos em março de 2019 (30,8\%) e 376 óbitos em março de 2020 (40,9\%). Observou-se que as taxas de óbito para o número total de atendimentos no SAMU nesses períodos foram 0,51 , 0,57 e 0,62 , respectivamente.

A Figura 1 mostra um aumento de 33\% nos casos de óbitos domiciliares entre março de 2018 e março de 2020. A Tabela 1 compara o número bruto de óbitos por PCR e outras causas, mostrando que 2020 teve mais notificações de óbitos domiciliares por PCR, com diferença estatística em relação a 2018 e 2019. 


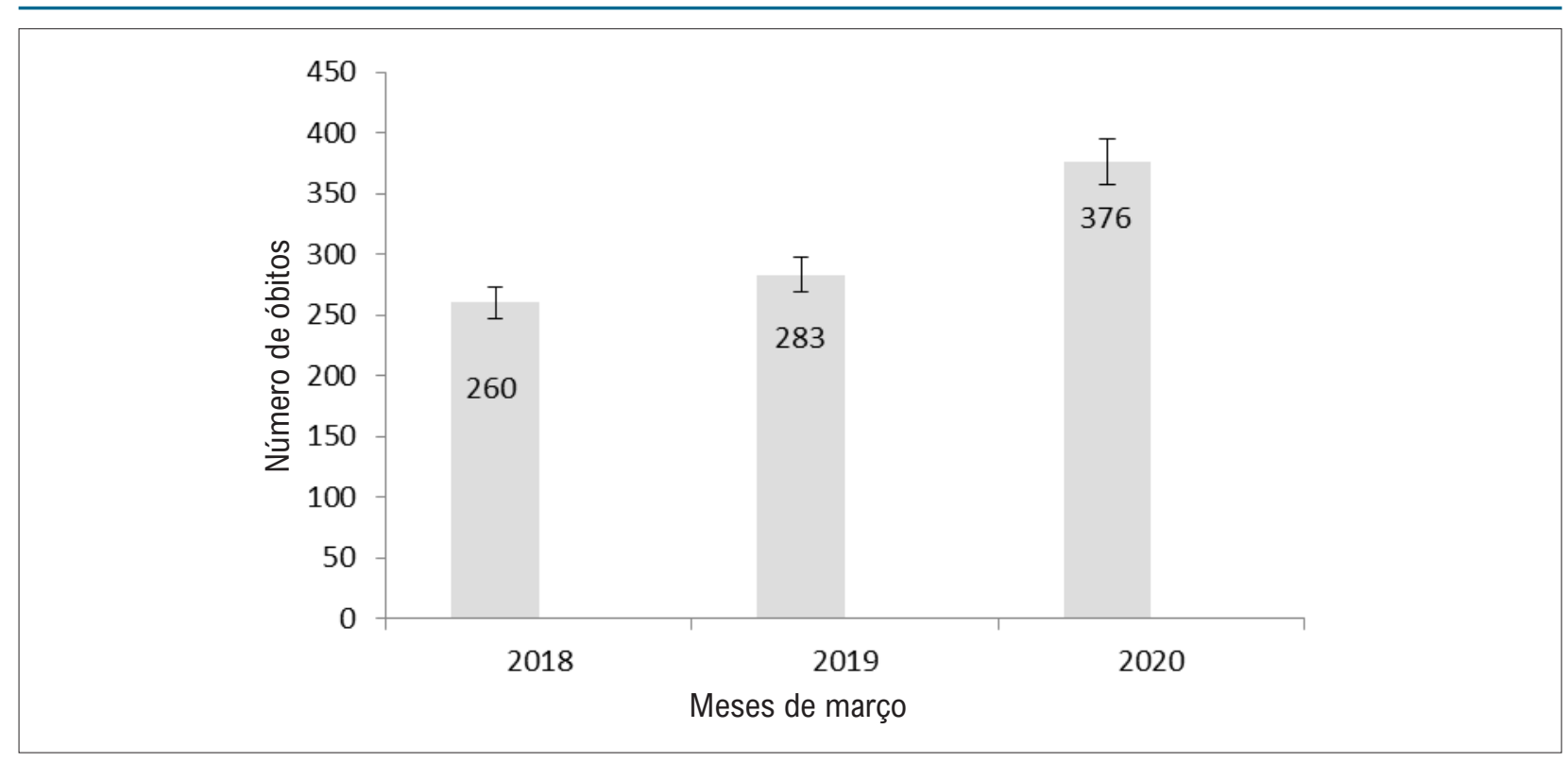

Figura 1 - Prevalência do desfecho de óbito em pacientes atendidos pelo Serviço de Atendimento Móvel de Urgência de Belo Horizonte, Minas Gerais, Brasil, Março 2018-2020.

Tabela 1 - Comparação de óbitos domiciliares por parada cardiorrespiratória e outras causas em março de 2018 e 2019 em relação a março de 2020, notificados pelo Serviço de Atendimento Móvel de Urgência de Belo Horizonte, Minas Gerais, Brasil

\begin{tabular}{|c|c|c|c|c|c|c|}
\hline \multirow[t]{2}{*}{ Óbitos } & \multicolumn{2}{|c|}{ Anos } & \multirow[t]{2}{*}{ Valor $\mathbf{p}^{*}$} & \multicolumn{2}{|c|}{ Anos } & \multirow[t]{2}{*}{ Valor $p$ * } \\
\hline & 2018 & 2020 & & 2019 & 2020 & \\
\hline PCR & 260 & 376 & $<0,01$ & 283 & 376 & 0,05 \\
\hline Outras causas & 303 & 229 & & 211 & 229 & \\
\hline
\end{tabular}

PCR: parada cardiorrespiratória; valor $p^{*}$ - teste qui-quadrado.

A Tabela 2 descreve as características sociais, clínicas e de notificação. Dos óbitos registrados em março de $2020,63,8 \%$ dos pacientes tinham 60 anos ou mais. De acordo com o horário de notificação, 63,7\% ocorreram no período diurno sendo $37,7 \%$ no período da manhã e $26,0 \%$ no período da tarde, sem diferença entre as proporções dos 31 dias consecutivos desse período (Tabela 2).

Em relação às características clínicas, observou-se que aproximadamente $87,0 \%$ dos pacientes com PCR apresentavam outras comorbidades clínicas e que a maioria da amostra avaliada não teve acompanhamento médico de acordo com os dados coletados $(88,7 \%)$ (Tabela 2). No entanto, muitos dos acompanhantes e familiares dos pacientes desconheciam o histórico dos pacientes.

A Tabela 3 descreve as comorbidades clínicas notificadas pelo serviço, que representam $87,0 \%$ ( $n=$ 331) dos pacientes avaliados, de acordo com a evolução da doença observada. Dentre as doenças crônicas observadas, a hipertensão esteve associada à PCR em $22,87 \%$ dos casos notificados; a insuficiência cardíaca estava presente em $13,03 \%$ dos casos e a diabetes mellitus em 11,0\%. Dentre outras comorbidades, é interessante notar que em $38,4 \%$ dos casos relatados, apesar de a família ou os amigos relatarem a presença de comorbidade associada, eles não souberam informar quais eram as comorbidades associadas do paciente.

\section{Discussão}

Como principal resultado deste estudo, observou-se aumento numérico gradativo da taxa de óbitos domiciliares por PCR para o total de atendimentos pelo SAMU e aumento proporcional de 33\% dos óbitos domiciliares em março de 2020, mês em que a Organização Mundial da Saúde declarou a pandemia de COVID-19. ${ }^{8}$

Desde a confirmação do primeiro caso no Brasil, em 26 de fevereiro de 2020, a imprensa, assim como as autoridades sanitárias, na ausência de vacinas ou medicamentos antivirais, alertam sobre a necessidade de distanciamento social, uso de máscaras, lavagem das mãos e reforço para cuidados com relação à etiqueta respiratória. Na cidade de Belo Horizonte, a primeira notificação de COVID-19 ocorreu em 16 de março de 2020; entretanto, desde a declaração mundial da pandemia, a prefeitura 
Tabela 2 - Análise descritiva de óbitos por parada cardiorrespiratória em março de 2020, notificados pelo Serviço de Atendimento Móvel de Urgência de Belo Horizonte, Minas Gerais, Brasil

\begin{tabular}{|c|c|c|}
\hline Variáveis & n & $\%$ \\
\hline \multicolumn{3}{|l|}{ Características sociais } \\
\hline \multicolumn{3}{|l|}{ Idade (anos) } \\
\hline$\leq 19$ & 10 & 2,70 \\
\hline $20-59$ & 126 & 33,50 \\
\hline$\geq 60$ & 240 & 63,80 \\
\hline \multicolumn{3}{|c|}{ Características de ocorrência de SAMU } \\
\hline \multicolumn{3}{|l|}{ Atendimento } \\
\hline Primeira metade de março & 190 & 50,50 \\
\hline Segunda metade de março & 186 & 49,50 \\
\hline \multicolumn{3}{|c|}{ Tempo de ocorrência de SAMU } \\
\hline $06: 00-11: 59$ & 142 & 37,70 \\
\hline $12: 00-17: 59$ & 98 & 26,00 \\
\hline $18: 00-23: 59$ & 89 & 23,60 \\
\hline $00: 00-05: 59$ & 47 & 12,70 \\
\hline \multicolumn{3}{|l|}{ Características clínicas } \\
\hline \multicolumn{3}{|l|}{ Causa de CPA } \\
\hline Clínica & 326 & 86,70 \\
\hline Trauma & 50 & 13,30 \\
\hline \multicolumn{3}{|l|}{ Acompanhamento médico } \\
\hline Sim & 45 & 12,00 \\
\hline Não & 331 & 88,00 \\
\hline
\end{tabular}

instituiu o isolamento social precocemente, evitando que os residentes tivessem contato não essencial.

Aproximadamente $80 \%$ dos casos de COVID-19 são leves ou oligossintomáticos. ${ }^{9} \mathrm{Um}$ estudo recente mostra que $20 \%$ de pacientes necessitaram de cuidados hospitalares, com $5 \%$ a $15 \%$ destes sendo tratados em unidades de terapia intensiva com necessidade de suporte ventilatório. A mortalidade para estes pacientes pode chegar a $80 \% .{ }^{9} \mathrm{O}$ SARS-CoV-2 pode ser transmitido de pessoa para pessoa (contato com as mãos, tosse, ou gotículas de saliva) ou através de superfícies e objetos contaminados pelo vírus. ${ }^{10}$ Em 16 de maio de 2020, mais de 4.605 .673 pessoas foram diagnosticadas com COVID-19 e houve 310.180 mortes ao redor do mundo. No Brasil, até 16 de maio de 2020, foram registrados 222.877 casos, com 15.046 óbitos. ${ }^{11}$ Dentre as medidas de prevenção da infecção pela COVID-19, recomenda-se aos indivíduos a higienização das mãos, superfícies e objetos com água e sabão ou com desinfetante para as mãos. Outras indicações incluem evitar o contato
Tabela 3 - Comorbidades relacionadas a óbitos por parada cardiorrespiratória em março de 2020 , notificados pelo Serviço de Atendimento Móvel de Urgência de Belo Horizonte, Minas Gerais, Brasil

\begin{tabular}{lcc}
\hline Comorbidades clínicas & $\mathbf{n}$ & $\%$ \\
\hline Não relatadas & 145 & 38,56 \\
\hline Hipertensão arterial & 86 & 22,87 \\
\hline Insuficiência cardíaca & 49 & 13,03 \\
\hline Diabetes mellitus & 42 & 11,17 \\
\hline Câncer & 40 & 10,64 \\
\hline Demência & 26 & 6,91 \\
\hline Infecções respiratórias & 23 & 6,12 \\
\hline Acidente vascular cerebral & 19 & 5,05 \\
\hline Arritmia & 12 & 3,19 \\
\hline Infecção do trato urinário & 3 & 0,80 \\
\hline
\end{tabular}

Tamanho da amostra $=376$.

com os olhos, nariz e boca; o uso permanente de máscara facial; a manutenção de hábitos saudáveis de alimentação e sono; e o isolamento e distanciamento social. ${ }^{12}$

O aumento do número de mortes, no contexto da pandemia, pode agravar o medo dos usuários de sair do isolamento social para buscar assistência médica e serviços essenciais. Isso poderia atrasar a demanda por serviços de saúde que afetam a doença subjacente. É interessante notar que quase $89 \%$ da nossa amostra não teve acompanhamento médico; em frequência semelhante (87\%), a natureza da PCR foi a causa clínica.

Em um estudo recente, Gonzales-Olmo et al.,13 com dados da população de Madrid, demonstrou elevados níveis de auto-percepção individual de maior vulnerabilidade em relação à infecção pela COVID-19 ao procurar atendimento odontológico, considerado um serviço essencial de saúde. Assim, os pesquisadores observaram que a amostra de indivíduos com mais de 60 anos com doenças sistêmicas evitou o atendimento odontológico na maioria das vezes. ${ }^{13}$

De acordo com os nossos resultados, Holmes et al. (2020) indicaram que houve uma quebra estrutural nas séries temporais de internações semanais diferidas anualmente de acordo com os serviços de emergência no Reino Unido entre setembro de 2019 e abril de 2020. ${ }^{14}$ Esses pesquisadores observaram o período de tempo correspondente a setembro de 2019 e abril de 2020 .

Foram desenvolvidas e estão em fase de validação escalas para avaliar o medo dos indivíduos (como a "Escala de Medo de COVID-19") com a finalidade de avaliar essa emoção. Espera-se que novos estudos avaliem o contexto da pandemia a esse respeito, monitorando o medo em detrimento de não procurar serviços essenciais de saúde, melhorando a qualidade de vida e retardando a mortalidade por causas que não envolvem COVID-19. ${ }^{15-17}$ 
Julia et al., ${ }^{18}$ relatam a reorganização do serviço de saúde na França durante a pandemia. Importantes investimentos têm sido feitos em termos de teleconsultas para acompanhamento de pacientes com COVID-19, mas a população mais vulnerável com dificuldades de acesso à internet e tecnologias digitais ou com barreiras linguísticas permaneceu sem assistência adequada. Além disso, os leitos hospitalares disponíveis foram drasticamente reduzidos e os profissionais de atenção primária à saúde consequentemente tiveram que lidar com emergências por doenças crônicas. ${ }^{18}$ As visitas domiciliares têm sido divididas por pacientes com e sem COVID-19, levando à sobrecarga do serviço. Já foi adotado um fluxo para a atenção básica aos pacientes com doenças crônicas, a fim de evitar atrasos no controle ambulatorial. ${ }^{17}$

Em Belo Horizonte, houve redução da demanda da população por atendimento nas Unidades Básicas de Saúde e Unidades de Pronto Atendimento. Durante os primeiros quatro meses de 2019, foram atendidas 1.478 .905 pessoas; no mesmo período de 2020, esse número foi de 1.215.543, o que representa uma redução de $18 \% .^{19}$ Dessa maneira, é importante monitorar o manejo das doenças crônicas e promover ações educativas para que a população compreenda a importância do acompanhamento da saúde e do uso regular dos medicamentos necessários. Dessa forma, a Secretaria Municipal de Saúde de Belo Horizonte atualizou o fluxo de atenção na atenção básica. ${ }^{19,20}$

Souza et al., ${ }^{21}$ relatam sobre a atenção primária à saúde prestada pelo SUS no Brasil e sobre os investimentos que vêm sendo destinados à aquisição de equipamentos e à ampliação de leitos hospitalares para pacientes com COVID-19. Reforçam a necessidade de fortalecer a atenção básica como instrumento de prevenção do colapso do sistema de saúde, evitando óbitos por COVID-19 e doenças crônicas. ${ }^{21}$ É necessário refletir sobre como encontrar o equilíbrio para que as ações de saúde não sejam paralisadas durante a pandemia.

A Secretaria Municipal de Saúde de Belo Horizonte tem realizado ações como treinamentos por meio de conferências virtuais, aulas virtuais, discussão de casos clínicos e notas técnicas para atendimento e fluxo de pacientes com COVID-19, estabelecendo parcerias para atendimento online e atendimento a pacientes de grupos de risco. Paralelamente, vem adotando medidas de adequação dos serviços de urgência e atenção primária à saúde, buscando não sobrecarregar os serviços e promover melhor atendimento aos pacientes com e sem COVID-19. ${ }^{20}$

O aumento do número de óbitos domiciliares tem despertado a atenção de gestores de saúde, e a ideia é reforçar a importância do controle ambulatorial das doenças crônicas e esclarecer as medidas de segurança adotadas para a população quanto às complicações clínicas não relacionadas à COVID-19 durante a pandemia.

Este é um momento favorável para reinventar a relação entre os usuários e os profissionais da atenção básica, buscando maior aproximação para o estabelecimento de vínculos, fortalecendo a importância do controle das doenças crônicas e evitando mortes desnecessárias. Também é necessário implementar medidas para melhorar a vigilância de óbitos. Os serviços de atenção primária à saúde devem organizar a assistência e os fluxos de, pacientes a fim de garantir o atendimento adequado aos pacientes com e sem COVID-19.

Como limitação do estudo, apontamos o alto percentual de indivíduos para os quais não foi possível coletar informações sobre a existência de comorbidades (38,5\% da amostra) devido à falta de informações dos familiares ou amigos entrevistados, que podem ter subestimado os valores apresentados.

\section{Conclusão}

Os resultados indicam um aumento no número de óbitos domiciliares por PCR notificados pelo SAMU em março de 2020 em relação a março de 2018 e março de 2019 em Belo Horizonte, Minas Gerais. Será necessário realizar estudos futuros com delineamento longitudinal para monitorar o aumento da mortalidade de usuários do sistema de saúde e analisar suas relações causais para evitar óbitos por outras doenças que não envolvam a COVID-19.

\section{Contribuição dos Autores}

Concepção e desenho da pesquisa: Guimarães NS, Carvalho TML, Pinto JM, Lage R, Bernardes RM, Peres ASS, Raposo MA, Rodrigues VM, Melo MCB, Tupinambás U; Obtenção de dados: Carvalho TML, Pinto JM, Lage R, Bernardes RM, Peres ASS, Raposo MA, Carvalhais RM, Shiomatsu GY, Oliveira BC, Melo MCB, Tupinambás U, Marcini RA; Análise e interpretação dos dados e Revisão crítica do manuscrito quanto ao conteúdo intelectual importante: Guimarães NS, Carvalho TML, Pinto JM, Lage R, Bernardes RM, Peres ASS, Raposo MA, Carvalhais RM, Shiomatsu GY, Oliveira BC, Rodrigues VM, Melo MCB, Tupinambás U; Análise estatística: Guimarães NS, Raposo MA, Tupinambás U; Redação do manuscrito: Guimarães NS, Carvalho TML, Pinto JM, Lage R, Bernardes RM, Peres ASS, Carvalhais RM, Shiomatsu GY, Oliveira BC, Rodrigues VM, Melo MCB, Tupinambás U, Marcini RA.

\section{Potencial Conflito de Interesses}

Declaramos não haver conflito de interesses pertinentes.

\section{Fontes de Financiamento}

O presente estudo não teve fontes de financiamento externas.

\section{Vinculação Acadêmica}

Não há vinculação deste estudo a programas de pósgraduação. 


\section{Referências}

1. Brasil. Ministério da Saúde. Portaria 1.600 de 7 de julho de 2011. Diário Oficial da União. Brasília, 2011.

2. O'Dwyer G, Konder MT, Reciputti LP, Macedo C, Lopes MGM. O processo de implantação do Serviço de Atendimento Móvel de Urgência no Brasil: estratégias de ação e dimensões estruturais. Cad Saúde Pública. 2017; 33 (7):1-14.

3. Brasil. Ministério da Saúde. Política Nacional de Atenção às Urgências e Rede de Atenção às Urgências. Portaria de Consolidação no 3, de 28 de setembro de 2017; consolida as normas sobre as redes do Sistema Único de Saúde. Brasília, 2017.

4. Sociedade Brasileira de Cardiologia. Cardiomêtro: Mortes por Doenças Cardiovasculares no Brasil. Rio de Janeiro, 2020. Disponível em: http://www. cardiometro.com.br/

5. Sociedade Brasileira de Cardiologia. Atualização da Diretriz de Ressuscitação Cardiopulmonar e Cuidados Cardiovasculares de Emergência da Sociedade Brasileira de Cardiologia - 2019. Arq Bras Cardiol .2019; 113(3):449-663.

6. Malta DC, França E, Abreu DMX, Perillo RD, Salmen MC, Teixeira RA et al. Mortalidade por doenças não transmissíveis no Brasil, 1990 a 2015, segundo estimativas do estudo de Carga Global de Doenças. São Paulo Med J. 2017; 135(3): 213-21.

7. Saltarelli RMF, Prado RR, Monteiro RA, Machado IE, Teixeira BSM, Malta DC. Mortes evitáveis por ações do Sistema Único de Saúde na população da Região Sudeste do Brasil. Ciênc Saúde Coletiva. 2019, 24(3):887-98.

8. World Health Organization. (WH))Naming the coronavirus disease (COVID-2019) and the virus that causes it. Geneva; 2020.

9. Wu Z, JenMcGoogan JM. Characteristics of and Important Lessons From the Coronavirus Disease 2019 (COVID-19) Outbreak in China Summary of a Report of 72314 Cases From the Chinese Center for Disease Control and Prevention. JAMA. 2020;323(13):1239-42.

10. Garg S, Kim L, Whitaker M et al. Hospitalization Rates and Characteristics of Patients Hospitalized with Laboratory-Confirmed Coronavirus Disease 2019 - COVID-NET, 14 States, March 1-30, 2020. MMWR Morb Mortal Wkly Rep. 2020 Abril 69(15):458-64.
11. Johns Hopkins University. Coronavirus Resourde Center. COVID-19 Global Cases by the Center for Systems Science and Engineering at John Hopkins University (JHCHSU). United States;2020New York; 2020.

12. Center for Disease Control and Prevention (CDC). How coronavirus spreads. Acessed in 2020 Apr 23] Available from: https://www.cdc.gov/ coronavirus/2019-ncov/prepare/transmission.html.

13. González-Olmo MJ, Ortega-MartínezAR, Delgado-Ramos B, Romero-Maroto M, Carrillo-Diaz M. Perceived vulnerability to coronavirus infection: impact on dental practice. Braz Oral Res. 2020;34:e044.

14. Holmes JL, Brake S, Docherty M, Lilford R, Watson S. Emergency ambulance services for heart attack and stroke during UK's COVID-19 lockdown. Lancet, 2020;395;May23.

15. Ahorsu DK, Lin CY, Imani V, Saffari M, Griffiths MD, Pakpour AH. The Fear of COVID-19 Scale: Development and Initial Validation. Int J Ment Health Addict. 2020; Mar 27:1-9.

16. oraci P, Ferrari A, Abbiati FA, Del Fante E, De Pace R, Urso A, et al. Validation and Psychometric Evaluation of the Italian Version of the Fear of COVID-19 Scale. Int J Ment Health Addict. 2020 May 4:1-10.

17. Bitan TH, Giron AG, Bloch Y, Mayer Y, Shiffman N, Mendlovic S. Fear of COVID-19 scale: Psychometric characteristics, reliability and validity in the Israeli population. Psychiatry Res. 2020;Jul;289:113100.

18. Julia C, Saynac Y, Le Joubioux C, Cailhol J, Lombrail P, Bouchaud O. Organising community primary care in the age of COVID-19: chanllenges in disadvantaged areas. Correspondence. Lancet Public Health. 2020;5(6):e313.

19. Prefeitura Municipal de Belo Horizonte. Secretaria Municipal de Saúde. Belo Horizonte. 2020. Nota técnica COVID-19. no 007/2020. CIEVS/GVIGE/ DPSV/GEAPS/GEURE/GERRE/GEASF/DIAS/GCINT/DMAC/SMSA/PBH. Belo Horizonte;2020.

20. Prefeitura Municipal Belo Horizonte. Secretaria Municipal de Saúde. CORONAVIRUS $=19$. Belo Horizonte;2020.

21. Souza CDF, Gois-Santos VT, Correia DS, Martins-Filho PR, Santos VS. The need to strengthen Primary Health Care in Brazil in the context of the COVID-19 pandemic. Braz Oral Res. 2020;34:e047. 